\title{
GM1 Ganglioside role in the interaction of Alpha-synuclein with lipid membranes: morphology and structure.
}

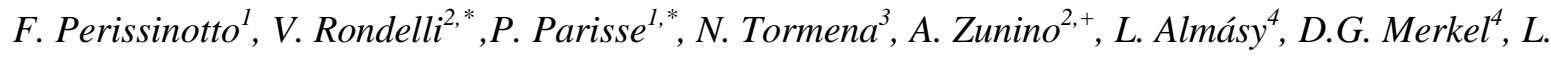 \\ Bottyán $^{4}$, Sz. Sajti ${ }^{4}$, L. Casalis ${ }^{1}$ \\ ${ }^{1}$ Elettra Sincrotrone Trieste S.C.p.A., Trieste, Italy \\ ${ }^{2}$ Department of Medical Biotechnologies and Translational Medicine, Università degli Studi di Milano, \\ Milano, Italy \\ ${ }^{3}$ Università degli Studi di Trieste, Trieste, Italy \\ ${ }^{4}$ Wigner Research Centre for Physics, Budapest, Hungary \\ *corresponding authors \\ ${ }^{+}$current address: Italian Institute of Technology, Genova, Italy
}

\begin{abstract}
Alpha-Synuclein (AS) is the protein playing the major role in Parkinson's disease (PD), a neurological disorder characterized by the degeneration of dopaminergic neurons and the accumulation of AS into amyloid plaques. The aggregation of AS into intermediate aggregates, called oligomers, and their pathological relation with biological membranes are considered key steps in the development and progression of the disease.

Here we propose a multi-technique approach to study the effects of AS in its monomeric and oligomeric forms on artificial lipid membranes containing GM1 ganglioside. GM1 is a component of functional membrane micro-domains, called lipid rafts, and has been demonstrated to bind AS in neurons. With the aim to understand the relation between gangliosides and AS, here we exploit the complementarity of microscopy (Atomic Force Microscopy) and neutron scattering (Small Angle Neutron Scattering and Neutron Reflectometry) techniques to analyze the structural changes of two different membranes (Phosphatidylcholine and Phosphatidylcholine/GM1) upon binding with AS. We observe the monomer- and oligomer-interactions are both limited to the external membrane leaflet and that the presence of ganglioside leads to a stronger interaction of the membranes and AS in its monomeric and oligomeric forms with a stronger aggressiveness in the latter. These results support the hypothesis of the critical role of lipid rafts not only in the biofunctioning of the protein, but even in the development and the progression of the Parkinson's disease.
\end{abstract}




\section{INTRODUCTION}

Alpha synuclein (AS) is an intrinsically disordered 140-amino acid protein highly expressed in the cytosol of neurons and primary involved in Parkinson's disease (PD) [1]. PD is a neurosynucleinopathy characterized by the accumulation of AS in fibrillar amyloid plaques in dopaminergic neurons of substantia nigra pars compacta (SNPC) [2].

Physiologically AS plays a crucial role in the homeostasis of synaptic vesicles interacting with proteins, such as SNARE family, which are the building blocks of the protein complex involved in neuronal exocytosis [3-5].

In its native state, AS is a monomer which binds to and assemble in alpha-helix conformation on lipid membranes [6-7]. Several studies performed on model membranes have demonstrated preferential binding of AS to negatively-charged phospholipids such as phosphatidylinositol (PI), phosphatidylglycerol (PG) and phosphatidylserine (PS) [8-12].

AS has been proposed to localize in vivo on specific plasma membrane domains, called lipid rafts, and this co-localization seems essential for the activity of the protein [13]. Lipid raft nano-domains have specific lipid composition, being enriched in sphingolipid and cholesterol, and have been implicated in several cell molecular pathways, some of which related to cancer, and other neurodegenerative diseases [14-16]. The situation is anyway controversial and binding of AS to lipid rafts is still under debate. It is still not clear whether AS prefers liquid ordered environment of lipid rafts, if the binding is mediated by its components (e.g. GM1 or GPI-anchored proteins) or this interaction is involved in the development of the pathology.

In pathological conditions, AS undergoes a misfolding structural rearrangement promoting the formation of toxic fibrillary aggregates in both intracellular and extracellular space in the brain [1719]. The misfolding and abnormal aggregation of AS is considered a key event in the development of PD. In the last decade studies have been increasingly focused on the intermediate products of AS aggregation, called oligomers. The oligomers, rather than amyloid fibrils are considered the major toxic species [20-22]. Many environmental and physicochemical factors, such as $\mathrm{pH}$, membraneinteraction, metal ions and anionic molecules can promote the formation of oligomers [23-25].

Among the different harmful effects on cell viability, oligomers have shown to induce neurotoxicity by cell membrane destabilization, via lipid extraction and pore formation [26-28], leading to alteration of calcium flux, membrane conductance and synaptic transmission [19], [29]. Therefore, the biological membrane plays a pivotal dual role: on the one hand as a physiological site of action of AS and on the other hand as a crucial target of the AS aggregates. 
Some experimental evidences have highlighted the role of (sialic acid-containing glycosphingolipids, GMs) in the binding of AS to lipid membranes. GMs are abundant in the lipid rafts of the nervous system [30]. Localization of AS with gangliosides, in particular with GM1 ganglioside, was found in a study using circular dichroism (CD) spectroscopy and molecular simulations [13]. The interaction of AS with GM1-containing artificial vesicles has shown to promote AS oligomers but prevent fibril formation [31]. Moreover, a neuroprotective role of GMs has been proposed [32]. Mice models deficient in GM1 have displayed a stronger accumulation of AS in nigral neurons [33, 34], and a consequent potential relevance in the progression of PD.

In this complex framework, we undertake a study on the interaction of AS with model membranes endowed with GM1 molecules (components of lipid rafts) in different aggregation states, i.e. monomers (physiological state) and oligomers (pathological form). We exploit the potentiality of atomic force microscopy (AFM) to obtain morphological information about molecular interactions at near-physiological conditions. We combine AFM measurements to small angle neutron scattering (SANS) and neutron reflectometry (NR) taking advantage of the sensitivity of neutrons to isotopes to obtain detailed structural information about the effects induced by AS on different model membrane systems [35], [36]. We demonstrate that GM1 gangliosides enhance binding of AS monomers to the lipid membrane suggesting an important role of GMs in the binding of AS to lipid rafts. Moreover, we show that in the presence of oligomers GM1 affects the type of membraneinteraction of the AS aggregates with the bilayer, indicating an active role of these molecules in the onset of PD.

\section{MATERIALS AND METHODS}

\section{Biological molecules and chemicals}

18:1 ( $\Delta$ 9-Cis) PC (1,2-dioleoyl-sn-glycero-3-phosphocholine, DOPC), 14:0 PC d54 (1,2dimyristoyl-d54-sn-glycero-3-phosphocholine, d ${ }_{54}$ DMPC), 16:0 PC d62 (1,2-dipalmitoyl-d62-snglycero-3-phosphocholine, $\mathrm{d}_{62}$ DPPC) and ganglioside GM1 (Brain, Ovine-Sodium Salt) have been purchased from Avanti polar Lipids (Alabaster, USA). Expression and purification of human wildtype Alpha synuclein (AS) was performed by the group of Prof. Giuseppe Legname (Laboratory of Prion Biology, SISSA - Scuola Internazionale Superiore di Studi Avanzati, Trieste, Italy) Briefly, the expression of the protein was performed into E. coli strain BL21 (DE3) transformed with a vector pET11a carrying the nucleotide sequence of human wild-type AS. Cells were grown in 100 $\mathrm{mg} / \mathrm{mL}$ ampicillin containing Luria-Bertani broth at $37{ }^{\circ} \mathrm{C}$ until an $\mathrm{A}_{600}$ of about 0.6 , followed by induction with $0.6 \mathrm{mM}$ isopropyl $\beta$-thiogalactopyranoside for 5 hours. The protein was successively 
extracted by the bacterial periplasm via osmotic-shock and purified by anion-exchange chromatography according to the method of Huang and colleagues [37]. Deuterium oxide (Heavy water, Water- $\mathrm{d}_{2}, \mathrm{D}_{2} \mathrm{O}$ ) has been purchased from Sigma Aldrich. $\mathrm{FeCl}_{2}$ solution was prepared by dissolving salt powder (Sigma Aldrich) in ultrapure $18.2 \mathrm{M} \Omega \cdot \mathrm{cm} \mathrm{H}_{2} \mathrm{O}$ (Milli-Q, Millipore SpA, Milan, Italy) and filtered with a sterile syringe-filter $(0.22 \mu \mathrm{m})$ prior to use.

\section{AS aggregation}

AS aggregation was performed by mixing $100 \mu \mathrm{g}$ of protein (final concentration: $35 \mu \mathrm{M}$ ) with 2 $\mathrm{mM} \mathrm{FeCl}{ }_{2}$. Protein solution was incubated at $37{ }^{\circ} \mathrm{C}$ under shaking for 1 hour. The products of aggregation were analyzed by AFM imaging. The aggregation induced by iron was also monitored by the Tioflavin T (ThT) assay. A total of $10 \mu \mathrm{M}$ ThT was added to each sample in a final working volume of $200 \mu \mathrm{L}$ per well. Fibril formation was monitored on either a GEMINI EM plate reader or a Spectramax M5 (Molecular Devices). Each condition (AS and AS $+\mathrm{FeCl}_{2}$ ) was run in triplicate, in 96 black well plates; each well containing 1 teflon bead. The plate was incubated at $37^{\circ} \mathrm{C}$, shaken, and ThT fluorescence readings were recorded every 5 min for 72 hours. For each assay run, the background fluorescence was also recorded.

\section{Lipid vesicle preparation for model membranes fabrication}

Lipid molecules were dissolved in the appropriate organic solvent (Chloroform for phospholipids and chloroform/methanol 2:1 for GM1) and combined in the appropriate ratios. Lipid solutions were deposited on round bottom boiling flasks and the organic solvent was let to evaporate under a gentle stream of nitrogen. Samples were placed under vacuum overnight in order to completely remove solvent residues and re-suspended in $150 \mathrm{mM} \mathrm{NaCl} \mathrm{D}_{2} \mathrm{O}$ buffer. Freeze-thawing method was applied to obtain multilamellar vesicles (MLVs) [38]: briefly, samples were treated with repetitive cycles $(n=5)$ of freezing in nitrogen solution and thawing in warm water. Then, MLVs were extruded 51 times through a polycarbonate membrane with $100 \mathrm{~nm}$ sized pores using a commercial extruder (Avanti Polar Lipids), to form small unilamellar vesicles (SUVs).

\section{Atomic force microscopy (AFM)}

AFM imaging was carried out on a commercial MFP-3D Stand Alone AFM microscope (from Asylum Research, Santa Barbara, CA) working at room temperature in dynamic AC-mode. Commercial silicon cantilevers (BL-AC40TS-C2, Olympus Micro Cantilevers, nominal spring k $0.09 \mathrm{nN} / \mathrm{nm}$ and OMCL-RC800PSA-1, Olympus Micro Cantilevers, nominal spring k $0.76 \mathrm{nN} / \mathrm{nm}$ ) have been chosen for imaging in liquid and air conditions, respectively. Images (of $2 \mu \mathrm{m} \times 2 \mu \mathrm{m}$ or 
$5 \mu \mathrm{m} \times 5 \mu \mathrm{m}$ size) were acquired in a $512 \times 512$ pixel block (with lateral resolution of 3.9 and 9.7 $\mathrm{nm}$, respectively) at a scan speed of 0.6-1.0 lines/s.

For AFM analysis of AS molecules, $10 \mu \mathrm{L}$ of each protein species solution at $0.35 \mu \mathrm{M}$ were spread onto $0.6 \times 0.6 \mathrm{~cm}^{2}$ piece of freshly cleaved mica, left to incubate for $1 \mathrm{~min}$, gently rinsed 3 times with Milli-Q $\mathrm{H}_{2} 0$, dried with a stream of nitrogen and imaged by AFM.

For AS membrane-interaction investigation we prepared two different model membranes: one made by DOPC phospholipid and one made by DOPC+GM1 ganglioside in a molar ratio of 10:0.5.

$100 \mu \mathrm{L}$ of SUVs solution $\left(0.5 \mathrm{mg} / \mathrm{mL}\right.$ in $\left.2 \mathrm{mM} \mathrm{CaCl}_{2}\right)$ of specific lipid composition were spotted on freshly cleaved mica attached to the AFM liquid chamber by ultrafast glue. The sample was left to incubate for 15 minutes at room temperature to promote vesicle adsorption, fusion and the formation of lipid bilayer on the surface. Then the lipid membrane was gently rinsed three times with Milli-Q $\mathrm{H}_{2} \mathrm{O}$ before AFM analysis to remove the excess of vesicles from the liquid sub-phase.

We used two different amounts of protein, $10 \mu \mathrm{g}$ and $100 \mu \mathrm{g}$ (final AS protein concentration of 450 $\mathrm{nM}$ and $4.5 \mu \mathrm{M}$ in the AFM liquid chamber, respectively) corresponding to a protein/lipid ratio (P/L) of 2:1 and 20:1. The protein was incubated to the planar lipid bilayer for 10 minutes and then imaged by AFM. AFM image analyses were performed using Gwyddion, an open-source modular program for scanning probe microscopy data visualization and analysis [39]. Data regarding size distribution of the AS molecules, the GM1-nanodomains and the AS aggregates on DOPC+GM1 bilayer were obtained using the grain analysis macro of the Gwyddion software. Graphs representing AFM trace profiles and height distributions were obtained using the Igor Pro software (Wavemetrics, US).

\section{Small angle neutron scattering (SANS) and neutron reflectometry (NR)}

While DMPC has been used as phospholipid matrix for the shorter SANS experiments, we used DPPC for NR, to ensure membrane stability during the long-lasting NR experiments. The use of deuterated phospholipids helps enhancing the visibility of the protein in interaction with the lipid bilayers, thanks to the induced membrane contrast change ( $c f$. Table $\mathrm{S} 1$ of the supporting information), the scattering length density of the lipids being around $7 \times 10^{-6} \AA^{-2}$, while that of proteins is around $2.5 \times 10^{-6} \AA^{-2}$.

The unpolarized beam NR measurements were carried out at the GINA neutron reflectometer at the Budapest Neutron Centre (BNC) [40], [41]. The membrane was measured at a neutron wavelength of $4.63 \AA$ in an aluminium liquid cell (temperature controlled by water circulation to $\pm 0.1^{\circ} \mathrm{C}$ ) which held a $70 \times 70 \times 10 \mathrm{~mm}^{3} \mathrm{Si}(100)$ block with an extremely smooth surface (rms roughness of $0.3 \mathrm{~nm}$ ) 
for membrane formation. The neutron beam entered the Si block through the $70 \times 10 \mathrm{~mm}$ face and was incident on the membrane from the Si block side.

The single supported $d_{62}$ DPPC membrane investigated by NR was diluted to the concentration of $0.5 \mathrm{mg} / \mathrm{mL}$ and deposited by vesicle fusion. Prior to the NR experiment, the Si block of the cell was cleaned by organic solvents, then washed with Milli-Q $\mathrm{H}_{2} \mathrm{O}$ and finally it was functionalized in a plasma cleaner for 15 minutes.

The SANS experiments were performed on the SANS-YS instrument at the Budapest Neutron Centre at two wavelengths and two sample-to-detector distances to cover a wide $q$-range, from 0.005 to $0.4 \AA^{-1}$. Vesicles at a concentration of $40 \mathrm{mg} / \mathrm{mL}$ were used for the SANS measurements and two model membrane systems $\left(\mathrm{d}_{54} \mathrm{DMPC}\right.$; and $\mathrm{d}_{54} \mathrm{DMPC}+\mathrm{GM} 1$ were investigated in a 10:1 molar ratio) a) alone (as prepared), b) in the presence of $\mathrm{Fe}(\mathrm{II})$ ions and c) in interaction with the protein at different states of aggregation (monomers and oligomers) to the final protein/lipid molar ratio 2:1. Samples, placed in quartz cells (produced by Hellma) were measured at $37^{\circ} \mathrm{C}$, above the melting temperature of the lipid chains.

\section{RESULTS AND DISCUSSION}

\section{AFM measurements of Alpha synuclein molecules}

Prior to investigate the membrane-interaction of human wild-type AS) in different aggregation states, monomeric $v s$ oligomeric, we performed a morphological analysis of both protein molecules via atomic force microscopy. As detailed in the Materials and Methods section, AS was spread onto freshly cleaved mica and analyzed in dry condition in dynamic mode. Imaging of monomeric AS showed the presence of globular spots with a height distribution in the range of $0.3-0.8 \mathrm{~nm}$ (Fig. 1 A), confirming the presence of both monomers and dimers, in agreement with previous experiments via scanning probe microscopy [42]. A comparative analysis was made with AS after treatment with $\mathrm{Fe}(\mathrm{II})$ ions.

We focused on the iron-mediated aggregation of AS because both in vivo and in vitro evidences of strong correlation between iron accumulation and AS aggregation have been reported in PD [4345]. Our approach was to investigate the aggregation of AS in pathological conditions, at high levels of iron, and compare the membrane-binding of the protein in different artificial model membranes. AS was first incubated in vitro with a high concentration iron salt solution for $1 \mathrm{~h}$ at 37 ${ }^{\circ} \mathrm{C}$ under shaking condition. AFM imaging revealed a fast aggregation induced by iron, with the formation of globular protein aggregates, which resemble oligomers (Fig. 1 B). These protein particles exhibit vertical dimensions in the range of $2.0-6.0 \mathrm{~nm}$ in agreement with a dimensional 
range of previous characterization of AS oligomeric species [43-45]. Prolonged incubation of the protein with iron did not promote fibrillar aggregates, suggesting that they could belong to an aggregation process different from fibril formation ( $c f$. ThT assay reported in the Supporting Information).

A
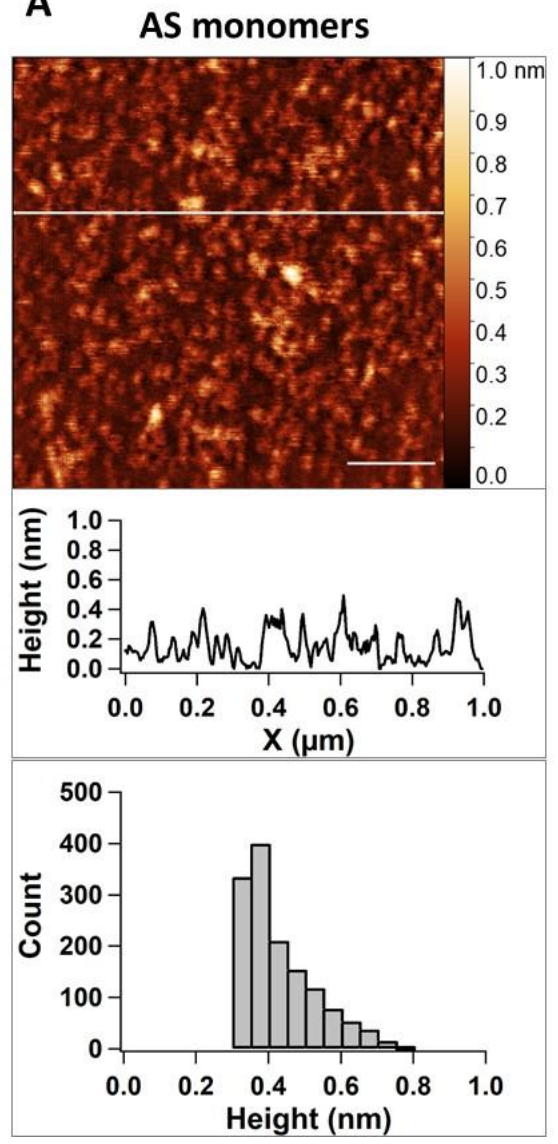

B AS oligomers
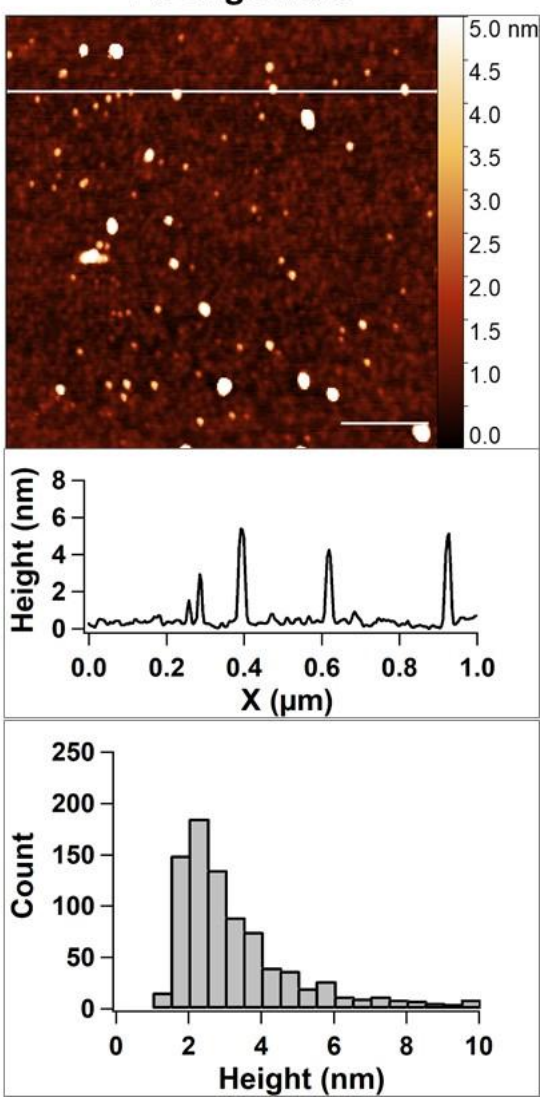

Figure 1. AFM imaging of human wild-type AS molecules. (A) AFM morphology of AS monomers and (B) AS oligomers with the relative section analysis profiles and distribution of heights. Proteins were spotted on freshly cleaved mica and analyzed in dynamic mode in dry conditions. Scale bar: $200 \mathbf{~ n m}$. Height distribution of monomers and oligomers were calculated from $n=5$ images.

\section{Effect of GM1 on the interaction of AS with supported lipid bilayers}

\section{AFM measurements}

To assess the effect of GM1 on AS-membrane binding, we produced two planar model lipid systems: a one-component phospholipid membrane made by DOPC and a two-component lipid bilayer containing DOPC and GM1 molecules at a molar ratio of 10:0.5. These systems were then analyzed via AFM dynamic imaging in liquid environment. 
AFM topographic analysis of membranes of DOPC alone showed a very flat and clean lipid surface with the presence of rare lipid defects represented by dark regions (Fig. 2 A). These defects have a depth of $2.0 \mathrm{~nm}$ which matches with the thickness of the upper monolayer of the membrane indicating that the defect is associated with the upper monolayer instead of the entire lipid bilayer. The analysis of different areas $(n>10)$ of the membrane showed less than $5 \%$ of lipid defects proving the good quality of the bilayer.

Similar to the DOPC bilayer, the membrane made by DOPC + GM1 exhibited high quality in terms of cleanliness and lipid coverage (>90\%). In addition, it displayed the presence of bright spots on the surface, which can be assigned to GM1 molecules assembling into nano-domains (Fig. 2 B). Most of these nano-islands have a height in the range $0.5-1.5 \mathrm{~nm}$ (Fig. 2 C). The bilayer displays rare defective areas which appear deeper than a monolayer, with a depth range corresponding to that of a complete lipid bilayer (around $4.0 \mathrm{~nm}$ ). Extensive AFM and X-ray diffraction studies [49], [50] were reported on the propensity of GM1 gangliosides to partition into domains on model membranes formed by different PC phospholipids species. The height range of these domains were shown to range from 1.0 to $2.0 \mathrm{~nm}$, in line with our AFM data. Moreover, we found a few larger GM1-nanodomains of 2.0 to $3.0 \mathrm{~nm}$ size (Fig. 2 C). These values could be due to an electrostatic repulsion occurring between the negatively-charged silicon nitride AFM tip and the polar GM1 headgroups, an effect which may depend on the applied force and the AFM imaging conditions [51].
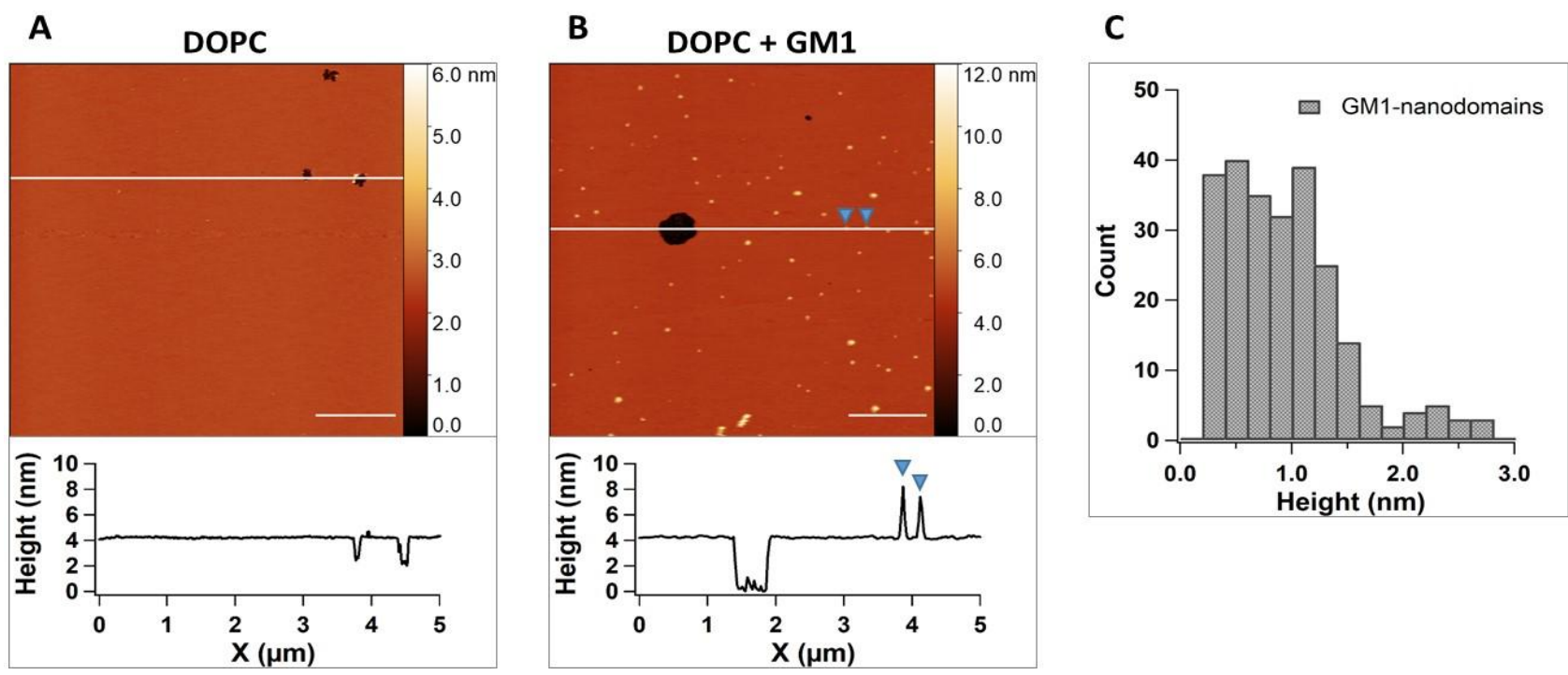

Figure 2. AFM topography of model membrane systems. (A) DOPC and (B) DOPC + GM1 (10:0.5 molar ratio) membranes with respective trace profiles along the surface. Blue inverted triangles indicate the nano-domains formed by GM1 molecules in the bilayer. Images were taken in liquid solution in dynamic mode. Scale bar: $1.0 \mu \mathrm{m}$. (C) Height distribution of GM1-domains calculated taken from multiple images $(n=5)$. 
We proceeded then to investigate the two model membrane systems, with and without GM1, in interaction with AS monomers / oligomers. We analyzed the binding of AS to the bilayer using two different protein concentrations (450 $\mathrm{nM}$ and $4.5 \mu \mathrm{M})$, corresponding to a protein/lipid ratio (P/L) of 2:1 and 20:1 respectively. AS-phenotypic effects on membrane have in fact been shown to dramatically depend on the protein concentration and the $\mathrm{P} / \mathrm{L}$ ratio [49-51]. The chosen concentrations are very close to physiological conditions, the AS being present in neurons in the low-micromolar concentration range [55].

We incubated AS with the model membranes and monitored the interaction via AFM. The monomeric form of $\mathrm{AS}$ at a P/L ratio of 2:1 showed a poor interaction with the DOPC bilayer leading to the formation of defect sites with a depth range of about 1.0-1.5 nm (Fig. $3 \mathrm{~A})$. This can be associated with the AS intercalation in the upper leaflet of the bilayer (one lipid layer is around $2.5 \mathrm{~nm}$ thick). The increase of the $\mathrm{P} / \mathrm{L}$ ratio to $20: 1$ leads to the formation of larger islands characterized by heights of about $2.0 \mathrm{~nm}$ (Fig. $3 \mathrm{~B}$ ). Since the thickness of fully-hydrated DOPC bilayer is around $5.0 \mathrm{~nm}$ [56], we assumed that monomeric AS is able to remodel the bilayer morphology intercalating in the upper lipid monolayer and leading to a thinning and lateral expansion of the lipid molecules. This mechanism has been speculated in other experimental studies on the interaction of AS monomer, as well as of antimicrobial peptides [54-56], with both planar and vesicular model lipid membranes of different lipid composition [57-60]. Moreover, membrane thinning processes have been associated with biological processes mediated by AS, such as in vivo synaptic vesicle formation [4] and, in general, endocytosis [64], [65].

The incubation of AS monomers to DOPC bilayer containing GM1 molecules showed surprising results. At low AS concentration (P/L 2:1) we observed a stronger interaction of the protein with the bilayer, when compared to the membrane lacking GM1; in fact we can observe (Fig. 3 C) the formation of larger defect sites then those observed in Figure 3 A-B. Cross sectional analysis of these patches reveals defects with a depth of $0.5 \mathrm{~nm}$ and white structures with heights in the range of 0.4-1.0 nm. Since the height of AS monomer has been calculated to be around $0.5-1.0 \mathrm{~nm}$ in AFM liquid measurements according to its adopted globular or extended conformation [66], our results supports a scenario in which the protein first "sit" on the bilayer via GM1-interaction and then slowly penetrate in the upper layer of the membrane.

Further, isolated nano-protrusions previously attributed to GM1 (Fig. 2B) disappear upon protein addition, suggesting that AS-membrane interaction is driven and enhanced by the GM1 molecules. This hypothesis is confirmed by increasing P/L to 20:1; the bilayer was almost completely defective, characterized by AS intercalation, with deeper, large islands of about $2.0 \mathrm{~nm}$ height. Only 
few areas of the membrane were unaffected by AS interaction (Fig. 3 D). All in all, we observed a 10-fold increase of AS monomeric interaction with the bilayer in the presence of GM1 molecules compared to the DOPC bilayer. These results evidence an active role of GM1 in mediating and strongly enhancing the interaction of AS with lipid membrane. We proved for the first time a direct and GM1-mediated increased binding of AS to an artificial lipid system. GM1 being a marker for lipid rafts in the cell membrane, the result is in agreement with the results of a previous study which reported a co-localization of monomeric AS with lipid raft components in brain extracts [13].

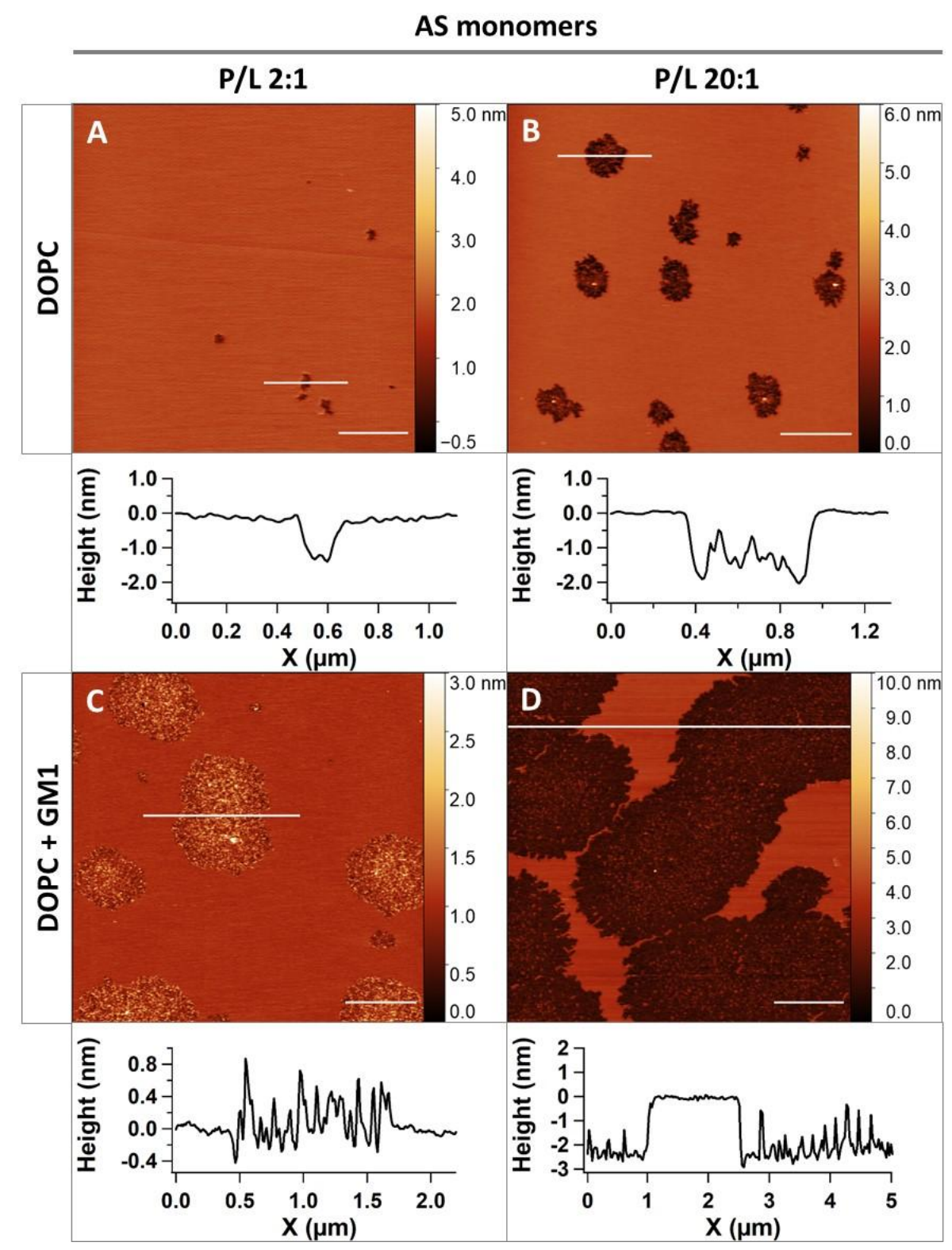

Figure 3. AFM measurements of monomeric AS interaction with DOPC and DOPC + GM1 model membranes. Evaluation of AS monomer binding and interaction with DOPC lipid bilayer at (A) $P / L=2: 1$ and (B) $P / L=20: 1$ protein/lipid ratio (P/L), respectively. (C-D) Equal experiments were performed for DOPC + GM1 membrane (10:0.5 molar ratio). Line profiles for each sample are shown under AFM images. In the line profile panels the height of the membrane was set to 0 , in order to 
emphasize the height variations due to the interacting proteins. Images were taken in liquid solution in dynamic mode. Scale bar: $1.0 \mu \mathrm{m}$.

The interaction of AS oligomers with model membranes DOPC and DOPC + GM1 were tested. The addition of protein aggregates at $\mathrm{P} / \mathrm{L}$ ratio $2: 1$ to the DOPC bilayer led to the formation of oligomeric clusters (Fig. 4 A) with a similar shape and lateral dimensions (0.2-1.0 $\mu \mathrm{m}$ ) of fractallike structures formed by the monomeric species at P/L 20:1 (Fig. 3 B), the main difference being in the much larger height, of about 5.0-10.0 nm. We can speculate of a similar membrane-interaction effect between monomers and oligomers with the difference that the aggregates species display a more aggressive behavior detectable already at low P/L ratio. Several mechanisms of interaction have been proposed for different oligomeric species involved in PD, such as pore formation and disruption of the bilayer [28], [63-65]. However, in our case the binding of oligomers does not seem to induce any of the mentioned effects, but seems to exhibit an interaction and/or protein accumulation in the upper monolayer of the membrane. When we increased P/L ratio to 20:1, surprisingly, we did not observe a significant increase in the lipid area covered by the clusters (except for a slight increase from $13 \%$ to 18\%). Moreover, we did not observe any other effect affecting the structural integrity of the membrane. On the contrary, the presence of GM1 drastically changed the interaction of oligomers with the lipid bilayer, now almost completely covered by protein particles (Fig. 4 B). The analysis of the heights showed a distribution very similar to the oligomers spotted on bare mica as shown in figure 1 B (Fig. 4 C). Also in this case GM1 seems to have played a major role in modulating the interaction of the AS oligomers with the bilayer. These results suggest that GM1 is able to prevent the formation of AS aggregated clusters on the lipid bilayer but seems to increase the membrane coverage of oligomers to the membrane, as in presence of a specific interaction with the ganglioside. This result could mean opposite effects in terms of toxicity in the pathology.

Currently, there is a general consensus on a neuroprotective function of GM1 in PD. GM1 has been shown to block the in vitro fibrillar aggregation of AS [31]. Moreover, a PD-mice model has displayed reduced levels of AS-aggregation and protection against loss of dopaminergic neurons after GM1-administering [32]. GM1 and the lipid rafts are also involved in other neurodegenerative disorders (Alzheimer's disease (AD) and Prion disorder (PD)), but the role seems to be different. GM1 gangliosides have been shown to promote enhanced toxic aggregation of the amyloid $\beta$ protein of AD [70]. Moreover, a GM1 interaction with the prion protein on the membrane of cell cultures seems to play an active role in the development of PD [71]. Considering these latter facts, a functional explanation of membrane-binding of AS in the presence of GM1 ganglioside, especially 
in the case of oligomeric AS, requires additional investigations in living systems (cell cultures, mice models).

\section{AS oligomers}

P/L 2:1

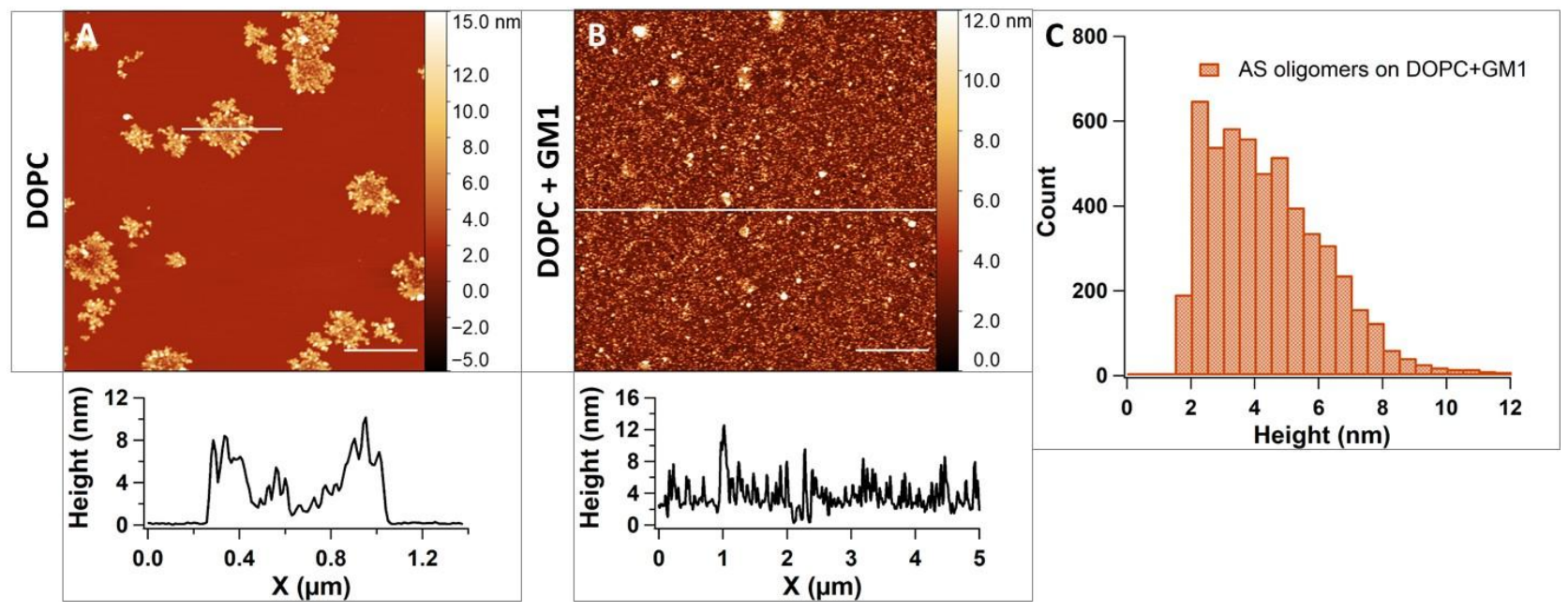

Figure 4. AFM measurements of AS oligomers-interaction with DOPC and DOPC + GM1 model membranes. (A) DOPC and (B) DOPC + GM1 bilayers after the addition of AS oligomers at P/L=2:1 protein/lipid ratio. Images were taken in liquid solution in dynamic mode. Scale bar: $1.0 \mu \mathrm{m}$. (C) Height distribution of protein particles on DOPC + GM1 bilayer.

\section{SANS measurements}

In order to obtain a better comprehension on the role of GM1 molecules in promoting AS binding to model membranes, we complemented AFM analysis with small angle neutron scattering (SANS) experiments on small unilamellar lipid vesicles (size $\sim 100 \mathrm{~nm}$ ) of similar lipid composition to the ones used for AFM experiments (DMPC and DMPC + GM1) - in presence of AS monomers and oligomers. In this set of experiments, we used deuterated DMPC phospholipid $\left(\mathrm{d}_{54} \mathrm{DMPC}\right)$, which is comparable to DOPC molecules, having the same lipid headgroup and being in fluid state at the working temperature. To avoid data misinterpretation, the effect of the Fe(II) on the model membranes has also been investigated by SANS ( $c f$. SI).

Figure 5 displays spectra collected on the DMPC + GM1 systems at $\mathrm{T}=37^{\circ} \mathrm{C}$. From the low $-q$ region of the spectra from we can gain information on the entire aggregates, i.e. the vesicles size. In this $q$-range no significant difference is visible between before and after the interaction of the membrane with the proteins. In the presence of iron a partial destabilization of the membrane was observed ( $c f$. SI), but this effect does not occur when AS oligomers are present in the solution, indicating that $\mathrm{Fe}$ (II) ions are strongly bound to the oligomers and are not released to the solvent 
and to the membranes. A significant effect is observed in the presence of AS oligomers. The SANS spectrum evidences the presence of large surface fractals sized more than hundred $\mathrm{nm}$ (which is the limit of the length scale investigated, that is $\sim 120 \mathrm{~nm}$ ). In fact, the slope of the profile approaching the lowest $q$-range shows a power low decrease with an exponent of -3.2 , typical of a surface fractal dimensionality [76]. In addition, the spectrum indicates the presence of $\sim 20 \mathrm{~nm}$ sized objects (evidenced by the broad peak found at $q \sim 0.01 \mathrm{~nm}^{-1}$ ), which may correspond to AS oligomers bound to the membrane, which otherwise would not be visible, giving a too low contribution to scattering because of their low total amount. The formation of large aggregates upon interaction may be associated with GM1 ganglioside inducing anchoring of AS oligomers to the membranes and promoting the formation of oligomer-based big aggregates extending in the extra membrane space. Alternatively, AS oligomers, having anchored to the GM1-containing membranes, act as linkers among membranes, promoting vesicles aggregation. The AFM measurements support this latter scenario.

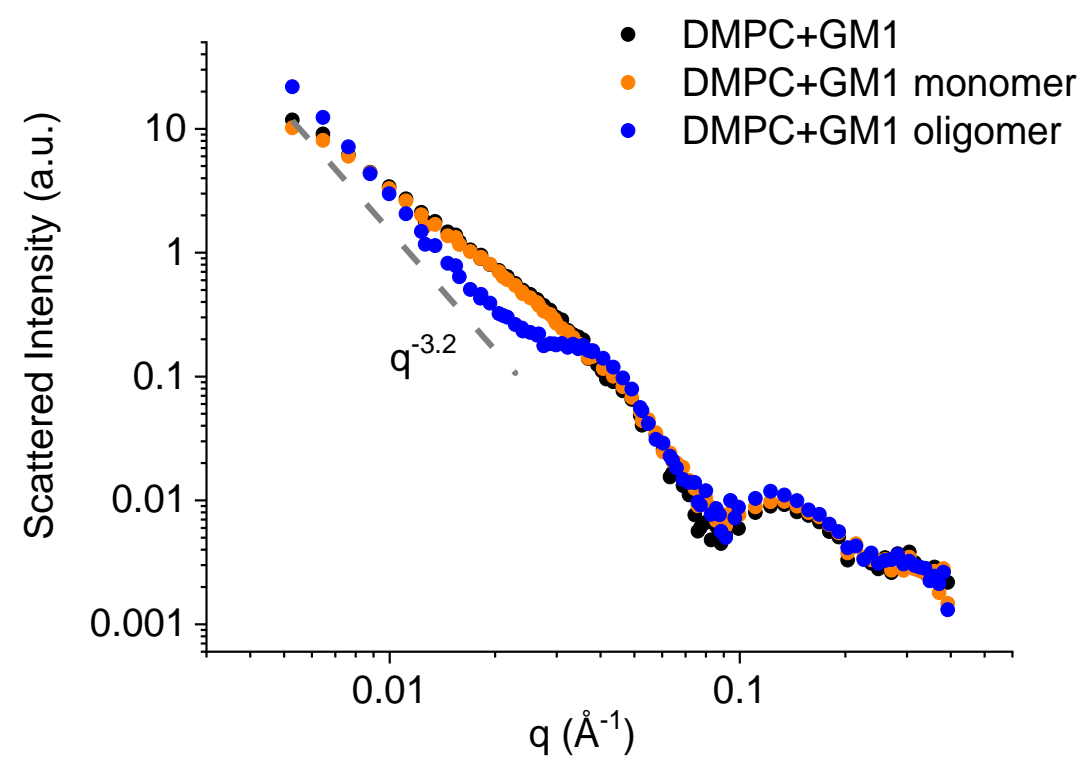

Figure 5. SANS spectra of the $\mathrm{d}_{54} \mathrm{DMPC}+\mathrm{GM1}$ membrane investigated alone (as prepared - black) and in interaction with the AS protein monomers (orange) or oligomers (blue). Measurements were performed at $\mathbf{T}=37^{\circ} \mathrm{C}$.

A core multi shell model has been fitted to SANS data of Fig. 5. with the SasView program [72], modeling the lipid vesicles as spherically shaped objects with a water core and formed by three contrasting layers: internal heads, deuterated chains and external heads. Each layer is characterized 
by its thickness and scattering length density (SLD). After having allowed for an interaction of AS with the bilayer, we did not observe any significant change in the SLD profile ( $c f$. SI and Fig. 6).

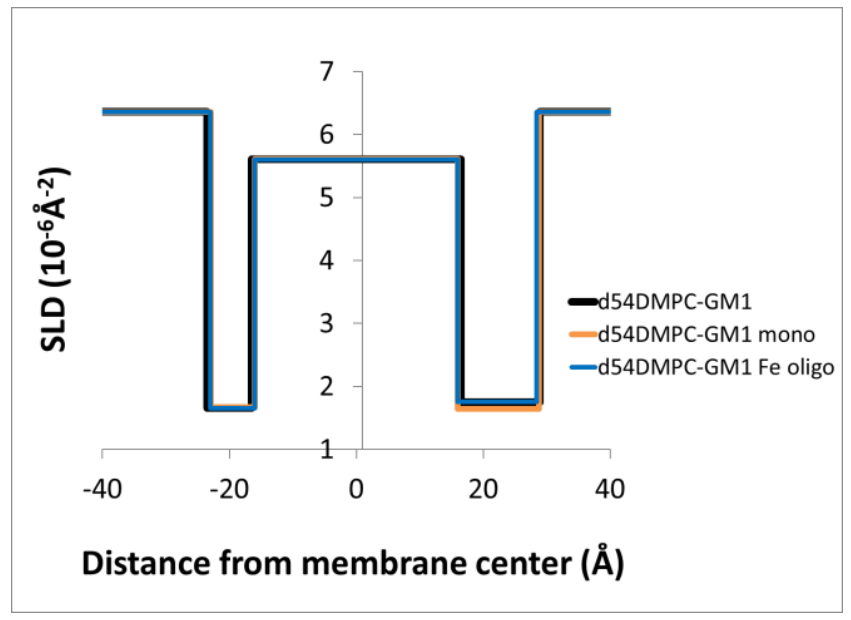

Figure 6. Scattering length density (SLD) profiles of $d_{54}$ DMPC + GM1 membrane in its as prepared state (black) and in interaction with the protein monomers (orange) as well as oligomers (blue), obtained by fitting a core-multi shell model to the SANS data.

On the one hand, when AS interacts with pure DMPC phospholipid vesicles, no significant difference is observed in the low- $q$ region upon the interaction with the protein, and no sensitive effect is detected in the presence of a small amount of Fe(II) added ( $c f$. SI). On the other hand, a spectral change is observed in the high- $q$ region, which is sensitive to the membrane form factor (Fig.7).

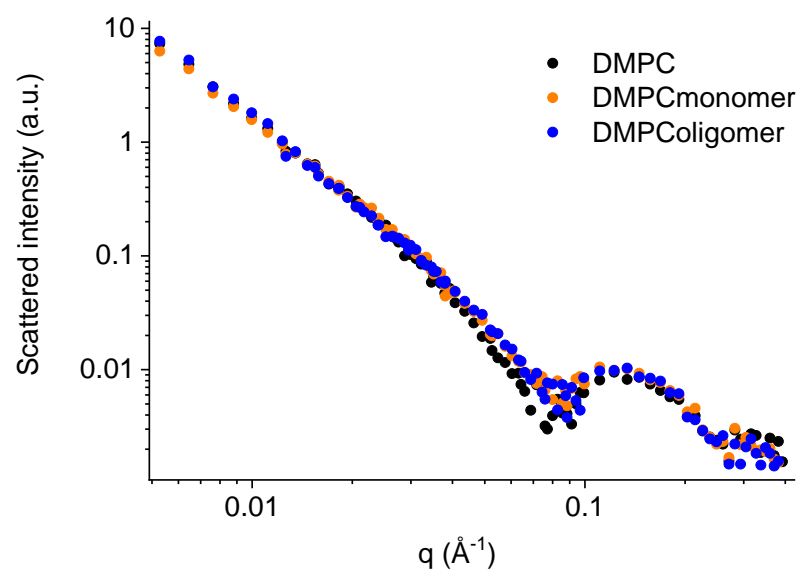

Figure 7 SANS spectra of $\mathbf{d}_{54}$ DMPC phospholipid membrane investigated alone, as prepared (black) and in interaction with the protein monomers (orange) and oligomers (blue). Measurements were performed at $\mathbf{T}=37^{\circ} \mathrm{C}$. 
A core multi shell model has also been fitted to the experimental data in Fig. 7.. The SLD profiles obtained for in the as prepared state and upon interaction with AS are shown in Figure 8 ( $c f$. Supporting Information for fit parameters). Results are consistent with a modification of the external heads layer, the contrast and thickness of which are affected by the presence of AS monomers and oligomers. Spectral changes are consistent with a thinning of the apolar and a thickening of the external polar region of the membrane, contrast of which is also increased, as if both AS monomers and oligomers interacted with the phospholipid membrane surface.

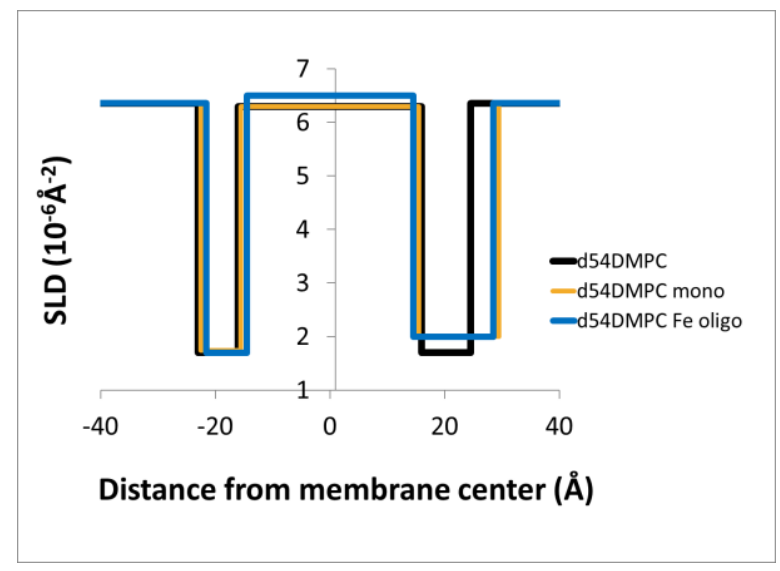

Figure 8. SLD profiles of the $d_{54}$ DMPC phospholipid membrane as prepared (black) and in interaction with the protein monomers (orange) and oligomers (blue), obtained by fitting a core-multi shell model to the SANS data.

\section{Neutron reflectometry}

To further study the protein-to-membrane interaction at a molecular scale, neutron reflectometry has been applied. NR is a suitable technique to unveil such details on the nanometer scale [35], [73]. Plefferkom and co-workers, by a combination of molecular dynamics and neutron reflection, validated the hypotesys that the interaction of AS monomers with a lipid membrane is limited to the external layer of the membrane [74], but similar investigations on the aggregated species of AS have not been performed to date.

To fill this gap we applied neutron reflectometry to investigate the cross structural changes caused by oligomeric AS aggregates to a single phospholipid membrane prepared by deuterated DPPC $\left(\mathrm{d}_{62}\right.$ DPPC). After silicon oxyde characterization (reflectivity measurement of the Si block surface with no membrane), a DPPC phospholipid membrane was deposited by vesicle fusion on the surface of the Si block and the NR was measured. Then the oligomer solution was injected directly in the measuring cell to reach the final P/L ratio of 2:1. 
After 1 hour of incubation NR was recorded, revealing that interaction occurred (Figure 9). In the following, another AS oligomers solution was injected into the cell, to reach the final P/L ratio of $4: 1$, to investigate whether interaction occured again and to what extent or the presence of AS species anchors the membrane surface (binds to the target membrane) which affects the interation. The final system was characterized in two contrasting solvents: $\mathrm{H}_{2} \mathrm{O}$ and $\mathrm{D}_{2} \mathrm{O}$. Data with the best fits and the resulting SLD profiles are reported in Figure 9. Analysis has been performed by the software MotoFit [75] accounting for a SLD of $2.5 \times 10^{-6} \AA^{-2}$ for the protein, while the structural results and the SLD valued used for lipids are reported in Table 1.

Results indicate that the membrane coverage was good (85\%). A water layer of $6 \AA$ thickness was found between the membrane and the silicon support. The interlayer membrane roughness was found to be around $3 \AA$. The NR data analysis confirmed the SANS results, unveiling that for AS monomers and oligomers alike the interaction with the membrane is limited to the outer layer of the lipid bilayer. Moreover, the interaction did not affect membrane coverage, i.e. the membrane stability. Data analysis also reveals that the extent of AS oligomers interaction with the phopsholipid membrane is slightly affected by the presence of preexistent aggregates. Increasing the quantity of incoming oligomers, in fact, we noted an increase of the penetration depth and a small increase in membrane's external layer thickness, compatible with an accumulation of protein on the membrane outer layer, in full accordance with AFM results reported in figure 4 A.
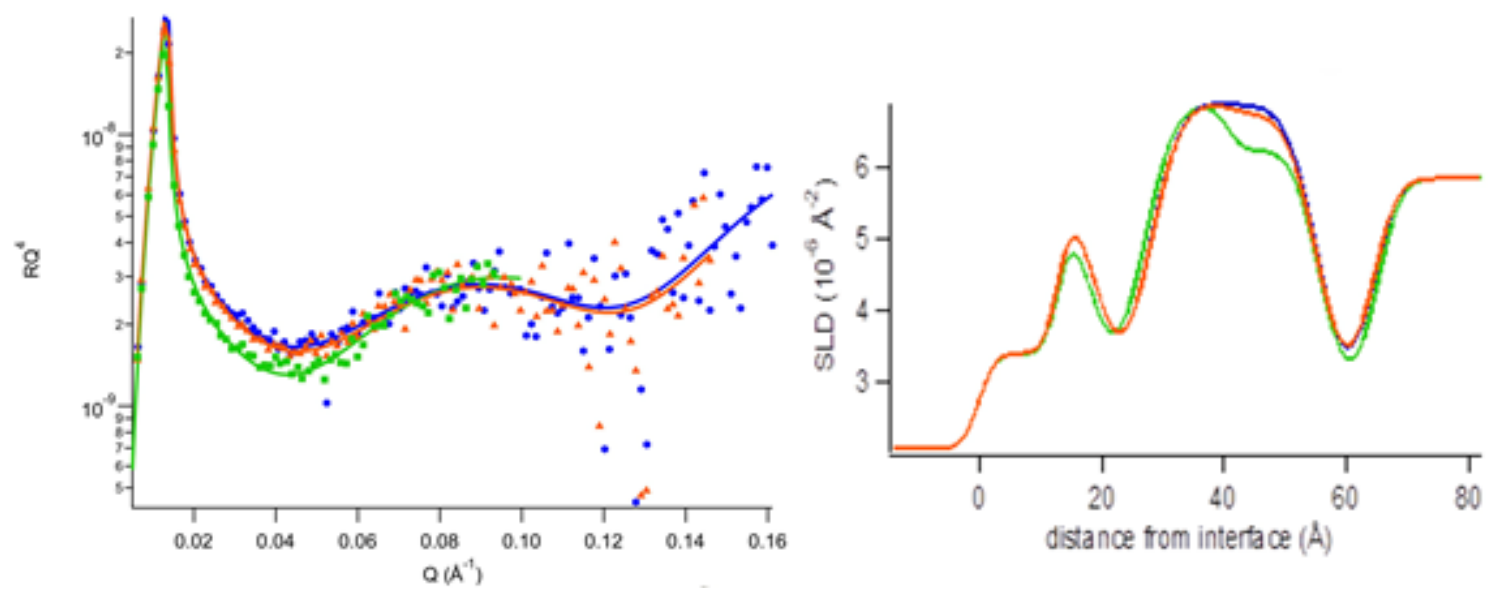

Figure 9. (A) Reflectivity curves and (B) SLD profiles obtained by the $d_{62}$ DPPC membrane in $D_{2} \mathrm{O}$ before and after the injection of AS oligomers. DPPC bilayer before (blue) and after AS oligomer incubation to the final P/L ratio of 2:1 (orange) and of 4:1 (green). Measurements were performed at $\mathrm{T}=45^{\circ} \mathrm{C}$, over the lipid melting temperature. 


\begin{tabular}{|c|ccc|cccc|cccc|}
\cline { 2 - 10 } \multicolumn{1}{c|}{} & \multicolumn{3}{c|}{ Membrane } & \multicolumn{4}{c|}{ Membrane + AS oligomers } & \multicolumn{3}{c|}{ Membrane + AS oligomers (x2) } \\
\cline { 2 - 11 } \multicolumn{1}{c|}{} & T & SLD & S & t & SLD & p & s & t & SLD & p & S \\
\hline Heads in & 9 & 1.75 & 25 & 9 & 1.75 & & 25 & 9 & 1.75 & & 25 \\
Chains in & 14 & 7.1 & 15 & 14 & 7.1 & & 15 & 14 & 7.1 & 15 \\
Chains out & 13 & 7.1 & 15 & 13 & $\mathbf{6 . 9}$ & $\mathbf{4 \%}$ & 15 & $\mathbf{1 5}$ & $\mathbf{6 . 3}$ & $\mathbf{1 7 \%}$ & 15 \\
Heads out & 9 & 1.75 & 25 & 9 & $\mathbf{1 . 8}$ & $\mathbf{7 \%}$ & 25 & $\mathbf{1 0}$ & $\mathbf{1 . 9}$ & $\mathbf{2 0 \%}$ & 25 \\
\hline
\end{tabular}

Table 1. Structural parameters of the $d_{62} \mathrm{DPPC}$ membrane investigated by NR before and after the interaction with AS oligomers. Abbreviations: $t=$ layer thickness $( \pm 1 \AA)$; SLD = layer SLD $( \pm 0.1 \times 10$ $\left.6 \AA^{-2}\right) ; p=$ estimated amount of protein inserted ( $\pm 3 \%$ volume); $s=$ layer solvent penetration $( \pm 5 \%$ volume). Normal numbers indicate membrane parameter values before, bold italics numbers indicate values after AS-membrane interaction.

\section{CONCLUSIONS}

With extended implications to the Parkinson's disease, a multi-techniques study was performed to clarify on the role of gangliosides in the interaction of artificial lipid membranes with alpha synuclein (AS) - in its monimeric and oligomeric form. We have shown that monomeric AS is able to remodel the membrane structure leading to bilayer thinning in both phospholipid and phospholipid containing GM1 membranes. The changes are not related to the type of AS-mediated effect on the bilayer but rather to the ability of GM1 molecules to enhance the membrane-binding of the AS, which supports speculations on an active role of the gangliosides in the subcellular localization of the protein. GM1 molecules enrich the raft domains of neuronal cell membranes, which affirm the hypothesis of a critical role of lipid rafts in the biological function of the protein. The iron-mediated AS oligomers have been demonstrated to behave similar to the monomers, except for their increased tendency in membrane binding. Even in this case, GM1 acts as a membrane-binding mediator, while triggering different binding routes of the aggregates with the membrane. The biological functionality of these interactions needs to be defined in living model systems.

In conclusion, we found evidences of crucial involvement of gangliosides, consequently of lipid rafts in the membrane-binding of AS in physiological as well as in pathological conditions.

\section{Acknowledgments}


We acknowledge CERIC-ERIC proposal grant n. 20167068 to perform measurements at the GINA and SANS-YS instruments of the Budapest Neutron Center. High purity functionalization of the Si block surface by the staff of the BioMEMS group of Inst. Tech. Physics and Mater. Sci., Budapest is gratefully acknowledged. FP, PP and LC acknowledge also the European Regional Development Fund and Interreg V-A Italia-Austria 2014-2020 project EXOTHERA (ITAT1036). We acknowledge Professor Giuseppe Legname and the Laboratory of Prion Biology (SISSA - Scuola Internazionale Superiore di Studi Avanzati, Trieste, Italy) for providing human Alpha synuclein protein. This research was funded by the Medical Biotechnologies and Translational Medicine Department of the Università degli Studi di Milano, grant number PSR2017 and PSR2018 assigned to V.R. This work benefited from the use of the SasView application, originally developed under NSF award DMR-0520547. SasView contains code developed with funding from the European Union's Horizon 2020 research and innovation programme under the SINE2020 project, grant agreement No 654000 .

\section{REFERENCES}

1. Goedert, M. Alpha-synuclein and neurodegenerative diseases. Nat. Rev. Neurosci. 2, 492501 (2001).

2. Spillantini, M. G. et al. $\alpha$-Synuclein in Lewy bodies. Nature 388, 839-840 (1997).

3. Burré, J. et al. $\alpha$-Synuclein Promotes SNARE-Complex Assembly in Vivo and in Vitro. Science. 329, 1663-1667 (2010).

4. Burré, J. The Synaptic Function of $\alpha$-Synuclein. J. Parkinsons. Dis. 5, 699-713 (2015).

5. Scott, D. \& Roy, S. $\alpha$-Synuclein Inhibits Intersynaptic Vesicle Mobility and Maintains Recycling-Pool Homeostasis. J. Neurosci. 32, 10129-10135 (2012).

6. Fusco, G. et al. Structural Ensembles of Membrane-bound $\alpha$-Synuclein Reveal the Molecular Determinants of Synaptic Vesicle Affinity. Sci. Rep. 6, 27125 (2016).

7. Ulmer, T. S., Bax, A., Cole, N. B. \& Nussbaum, R. L. Structure and Dynamics of Micellebound Human $\alpha$-Synuclein. J. Biol. Chem. 280, 9595-9603 (2005).

8. Middleton, E. R. \& Rhoades, E. Effects of Curvature and Composition on $\alpha$-Synuclein Binding to Lipid Vesicles. Biophys. J. 99, 2279-2288 (2010).

9. Davidson, W. S., Jonas, A., Clayton, D. F. \& George, J. M. Stabilization of alpha-synuclein secondary structure upon binding to synthetic membranes. J. Biol. Chem. 273, 9443-9 (1998).

10. Jo, E., McLaurin, J., Yip, C. M., St George-Hyslop, P. \& Fraser, P. E. alpha-Synuclein 
membrane interactions and lipid specificity. J. Biol. Chem. 275, 34328-34 (2000).

11. Pfefferkorn, C. M., Jiang, Z. \& Lee, J. C. Biophysics of $\alpha$-synuclein membrane interactions. Biochim. Biophys. Acta - Biomembr. 1818, 162-171 (2012).

12. Stöckl, M., Fischer, P., Wanker, E. \& Herrmann, A. $\alpha$-Synuclein Selectively Binds to Anionic Phospholipids Embedded in Liquid-Disordered Domains. J. Mol. Biol. 375, 1394 1404 (2008).

13. Fortin, D. L. et al. Lipid Rafts Mediate the Synaptic Localization of $\alpha$-Synuclein. $J$. Neurosci. 24, 6715-23 (2004):

14. Simons, K. \& Ikonen, E. Functional rafts in cell membranes. Nature 387, 569-572 (1997).

15. Sezgin, E., Levental, I., Mayor, S. \& Eggeling, C. The mystery of membrane organization: composition, regulation and roles of lipid rafts. Nat. Rev. Mol. Cell Biol. 18, 361-374 (2017).

16. Pike, L. J. Rafts defined: a report on the Keystone symposium on lipid rafts and cell function. J. Lipid Res. 47, 1597-1598 (2006).

17. Uversky, V. N. $\alpha$-Synuclein Aggregation and Parkinson's Disease. in Protein Misfolding, Aggregation, and Conformational Diseases. 61-110 (Springer, US, 2007)

18. Hoffmann, A. C. et al. Extracellular aggregated alpha synuclein primarily triggers lysosomal dysfunction in neural cells prevented by trehalose. Sci. Rep. 9, (2019).

19. Pacheco, C. R. et al. Extracellular $\alpha$-synuclein alters synaptic transmission in brain neurons by perforating the neuronal plasma membrane. J. Neurochem. 132, 731-741 (2015).

20. Winner, B. et al. In vivo demonstration that $\alpha$-synuclein oligomers are toxic. Proc. Natl. Acad. Sci. 108, 4194-4199 (2011).

21. Ingelsson, M. Alpha-Synuclein Oligomers-Neurotoxic Molecules in Parkinson's Disease and Other Lewy Body Disorders. Front. Neurosci. 10, 408 (2016).

22. Bengoa-Vergniory, N., Roberts, R. F., Wade-Martins, R. \& Alegre-Abarrategui, J. Alphasynuclein oligomers: a new hope. Acta Neuropathol. 134, 819-838 (2017).

23. Wu, K.-P., Weinstock, D. S., Narayanan, C., Levy, R. M. \& Baum, J. Structural Reorganization of $\alpha$-Synuclein at Low pH Observed by NMR and REMD Simulations. $J$. Mol. Biol. 391, 784-796 (2009).

24. Uversky, V. N., Li, J. \& Fink, A. L. Metal-triggered Structural Transformations, Aggregation, and Fibrillation of Human $\alpha$-Synuclein. J. Biol. Chem. 276, 44284-44296 (2001).

25. Lee, H.-J., Choi, C. \& Lee, S.-J. Membrane-bound alpha-synuclein has a high aggregation propensity and the ability to seed the aggregation of the cytosolic form. J. Biol. Chem. 277, $671-8$ (2002). 
26. Andreasen, M., Lorenzen, N. \& Otzen, D. Interactions between misfolded protein oligomers and membranes: A central topic in neurodegenerative diseases? Biochim. Biophys. Acta Biomembr. 1848, 1897-1907 (2015).

27. Stöckl, M., Claessens, M. M. A. E. \& Subramaniam, V. Kinetic measurements give new insights into lipid membrane permeabilization by $\alpha$-synuclein oligomers. Mol. BioSyst. 8 , 338-345 (2012).

28. Tosatto, L. et al. Alpha-synuclein pore forming activity upon membrane association. Biochim. Biophys. Acta - Biomembr. 1818, 2876-2883 (2012).

29. Danzer, K. M. et al. Different Species of $\alpha$-Synuclein Oligomers Induce Calcium Influx and Seeding. J. Neurosci. 27, 9220-9232 (2007).

30. Yu, R. K., Nakatani, Y. \& Yanagisawa, M. The role of glycosphingolipid metabolism in the developing brain. J. Lipid Res. 50, S440-S445 (2009).

31. Martinez, Z., Zhu, M., Han, S. \& Fink, A. L. GM1 Specifically Interacts with $\alpha$-Synuclein and Inhibits Fibrillation. Biochemistry. 46, 1868-77 (2017)

32. Schneider, J. S. et al. GM1 Ganglioside Modifies $\alpha$-Synuclein Toxicity and is Neuroprotective in a Rat $\alpha$-Synuclein Model of Parkinson's Disease. Sci. Rep. 9, 8362 (2019).

33. Wu, G., Lu, Z.-H., Kulkarni, N., Amin, R. \& Ledeen, R. W. Mice Lacking Major Brain Gangliosides Develop Parkinsonism. Neurochem. Res. 36, 1706-1714 (2011).

34. Forsayeth, J. \& Hadaczek, P. Ganglioside Metabolism and Parkinson's Disease. Front. Neurosci. 12, (2018).

35. Rondelli, V. et al. Amyloid- $\beta$ peptides in interaction with raft-mime model membranes: a neutron reflectivity insight. Sci. Rep. 6, 20997 (2016).

36. Fragneto, G. Neutrons and model membranes. Eur. Phys. J. Spec. Top. 213, 327-342 (2012).

37. Huang, C., Ren, G., Zhou, H. \& Wang, C. A new method for purification of recombinant human $\alpha$-synuclein in Escherichia coli. Protein Expr. Purif. 42, 173-177 (2005).

38. Rondelli, V. et al. Building a biomimetic membrane for neutron reflectivity investigation: Complexity, asymmetry and contrast. Biophys. Chem. 229, 135-141 (2017).

39. Nečas, D. \& Klapetek, P. Gwyddion: an open-source software for SPM data analysis. Open Phys. 10, 181-188 (2012).

40. Bottyán, L. et al. GINA-A polarized neutron reflectometer at the Budapest Neutron Centre. Rev. Sci. Instrum. 84, 015112 (2013).

41. Bottyán, L., Merkel, D. G., Nagy, B. \& Major, J. Neutron Reflectometer with Polarization Option at the Budapest Neutron Centre. Neutron News 23, 21-24 (2012). 
42. Ruggeri, F. S. et al. Identification and nanomechanical characterization of the fundamental single-strand protofilaments of amyloid $\alpha$-synuclein fibrils. Proc. Natl. Acad. Sci. 115, 72307235 (2018).

43. Hirsch, E. C., Brandel, J. P., Galle, P., Javoy-Agid, F. \& Agid, Y. Iron and aluminum increase in the substantia nigra of patients with Parkinson's disease: an X-ray microanalysis. J. Neurochem. 56, 446-51 (1991).

44. Ortega, R. et al. $\alpha$-Synuclein Over-Expression Induces Increased Iron Accumulation and Redistribution in Iron-Exposed Neurons. Mol. Neurobiol. 53, 1925-1934 (2016).

45. Ostrerova-Golts, N. et al. The A53T alpha-synuclein mutation increases iron-dependent aggregation and toxicity. J. Neurosci. 20, 6048-54 (2000).

46. Chen, S. W. et al. Structural characterization of toxic oligomers that are kinetically trapped during $\alpha$-synuclein fibril formation. Proc. Natl. Acad. Sci. 112, E1994-E2003 (2015).

47. Cremades, N., Chen, S. W. \& Dobson, C. M. Structural Characteristics of $\alpha$-Synuclein Oligomers. Int. Rev. Cell Mol. Biol. 329, 79-143 (2017).

48. Lashuel, H. A. et al. $\alpha$-Synuclein, Especially the Parkinson's Disease-associated Mutants, Forms Pore-like Annular and Tubular Protofibrils. J. Mol. Biol. 322, 1089-1102 (2002).

49. Yuan, C. \& Johnston, L. J. Atomic Force Microscopy Studies of Ganglioside GM1 Domains in Phosphatidylcholine and Phosphatidylcholine/Cholesterol Bilayers. Biophys. J. 81, 10591069 (2001).

50. McIntosh, T. J. \& Simon, S. A. Long- and Short-Range Interactions between Phospholipid/Ganglioside GM1 Bilayers. Biochemistry 33, 10477-10486 (1994).

51. Müller, D. J. \& Engel, A. The height of biomolecules measured with the atomic force microscope depends on electrostatic interactions. Biophys. J. 73, 1633-1644 (1997).

52. Galvagnion, C. et al. Lipid vesicles trigger $\alpha$-synuclein aggregation by stimulating primary nucleation. Nat. Chem. Biol. 11, 229-234 (2015).

53. Terakawa, M. S. et al. Membrane-induced initial structure of $\alpha$-synuclein control its amyloidogenesis on model membranes. Biochim. Biophys. Acta - Biomembr. 1860, 757-766 (2018).

54. Powers, R. et al. Metabolic Investigations of the Molecular Mechanisms Associated with Parkinson's Disease. Metabolites. 7, 22 (2017).

55. Borghi, R. et al. Full length alpha-synuclein is present in cerebrospinal fluid from Parkinson's disease and normal subjects. Neurosci. Lett. 287, 65-7 (2000).

56. Attwood, S., Choi, Y. \& Leonenko, Z. Preparation of DOPC and DPPC Supported Planar Lipid Bilayers for Atomic Force Microscopy and Atomic Force Spectroscopy. Int. J. Mol. 
Sci. 14, 3514-3539 (2013).

57. Hung, W.-C. et al. Membrane-thinning effect of curcumin. Biophys. J. 94, 4331-8 (2008).

58. Heath, G. R., Harrison, P. L., Strong, P. N., Evans, S. D. \& Miller, K. Visualization of diffusion limited antimicrobial peptide attack on supported lipid membranes. Soft Matter 14, 6146-6154 (2018).

59. Chen, F.-Y., Lee, M.-T. \& Huang, H. W. Evidence for membrane thinning effect as the mechanism for peptide-induced pore formation. Biophys. J. 84, 3751-8 (2003).

60. Giannakis, E. et al. Dimeric structures of $\alpha$-synuclein bind preferentially to lipid membranes. Biochim. Biophys. Acta - Biomembr. 1778, 1112-1119 (2008).

61. Mizuno, N. et al. Remodeling of Lipid Vesicles into Cylindrical Micelles by $\alpha$-Synuclein in an Extended $\alpha$-Helical Conformation. J. Biol. Chem. 287, 29301-29311 (2012).

62. Ouberai, M. M. et al. $\alpha$-Synuclein Senses Lipid Packing Defects and Induces Lateral Expansion of Lipids Leading to Membrane Remodeling. J. Biol. Chem. 288, 20883-20895 (2013).

63. Pan, J., Dalzini, A., Khadka, N. K., Aryal, C. M. \& Song, L. Lipid Extraction by $\alpha$-Synuclein Generates Semi-Transmembrane Defects and Lipoprotein Nanoparticles. ACS Omega 3, 9586-9597 (2018).

64. Szule, J. A. \& Coorssen, J. R. Revisiting the role of SNAREs in exocytosis and membrane fusion. Biochim. Biophys. Acta - Mol. Cell Res. 1641, 121-135 (2003).

65. Gauthier, N. C., Fardin, M. A., Roca-Cusachs, P. \& Sheetz, M. P. Temporary increase in plasma membrane tension coordinates the activation of exocytosis and contraction during cell spreading. Proc. Natl. Acad. Sci. U. S. A. 108, 14467-72 (2011).

66. Zhang, Y. et al. High-speed atomic force microscopy reveals structural dynamics of $\alpha$ synuclein monomers and dimers. J. Chem. Phys. 148, 123322 (2018).

67. Tsigelny, I. F. et al. Role of $\alpha$-synuclein penetration into the membrane in the mechanisms of oligomer pore formation. FEBS J. 279, 1000-1013 (2012).

68. Chaudhary, H., Iyer, A., Subramaniam, V. \& Claessens, M. M. A. E. $\alpha$-Synuclein Oligomers Stabilize Pre-Existing Defects in Supported Bilayers and Propagate Membrane Damage in a Fractal-Like Pattern. Langmuir 32, 11827-11836 (2016).

69. Dong, C. et al. Structural characteristics and membrane interactions of tandem $\alpha$-synuclein oligomers. Sci. Rep. 8, 6755 (2018).

70. Ikeda, K., Yamaguchi, T., Fukunaga, S., Hoshino, M. \& Matsuzaki, K. Mechanism of Amyloid $\beta$-Protein Aggregation Mediated by GM1 Ganglioside Clusters. Biochemistry 50, 6433-6440 (2011). 
71. Botto, L. et al. Role of Lipid Rafts and GM1 in the Segregation and Processing of Prion Protein. PLoS One 9, e98344 (2014).

72. SasView - Small Angle Scattering Analysis. Available at: https://www.sasview.org/. (Accessed: 29th June 2019)

73. Rondelli, V. et al. Ganglioside GM1 forces the redistribution of cholesterol in a biomimetic membrane. Biochim. Biophys. Acta - Biomembr. 1818, 2860-2867 (2012).

74. Pfefferkorn, C. M. et al. Depth of $\alpha$-synuclein in a bilayer determined by fluorescence, neutron reflectometry, and computation. Biophys. J. 102, 613-21 (2012).

75. Nelson, A. Co-refinement of multiple-contrast neutron/X-ray reflectivity data using MOTOFIT. J. Appl. Crystallogr. 39, 273-276 (2006).

76- Cherny A.Y., et al. Small-angle scattering from multiphase fractals. J. Phys. Conf. Ser. 393, 012031. (2013) 


\section{Supporting Material for the manuscript:}

GM1 Ganglioside role in the interaction of Alpha-synuclein with lipid membranes: morphology and structure.

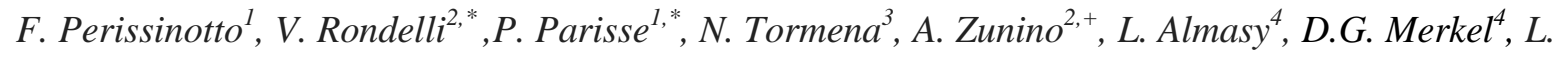
Bottyan $^{4}, \mathrm{Sz} . \mathrm{Sajti}^{4}$, L. Casalis ${ }^{1}$

${ }^{1}$ Elettra Sincrotrone Trieste S.C.p.A., Trieste, Italy

${ }^{2}$ Department of Medical Biotechnologies and Translational Medicine, Università degli Studi di Milano, Milano, Italy

${ }^{3}$ Università degli Studi di Trieste, Trieste, Italy

${ }^{4}$ Wigner Research Centre for Physics, Hungarian Academy of Sciences, Budapest, Hungary * corresponding authors

${ }^{+}$current address: Italian Institute of Technology, Genova, Italy

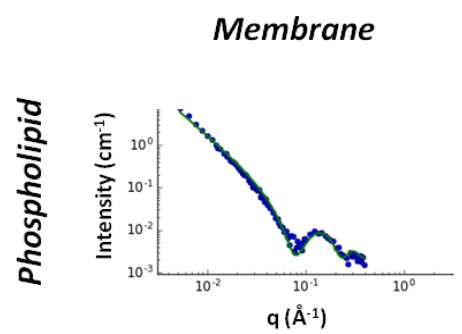

Membrane + Fe

Membrane + monomer

Membrane + oligomer
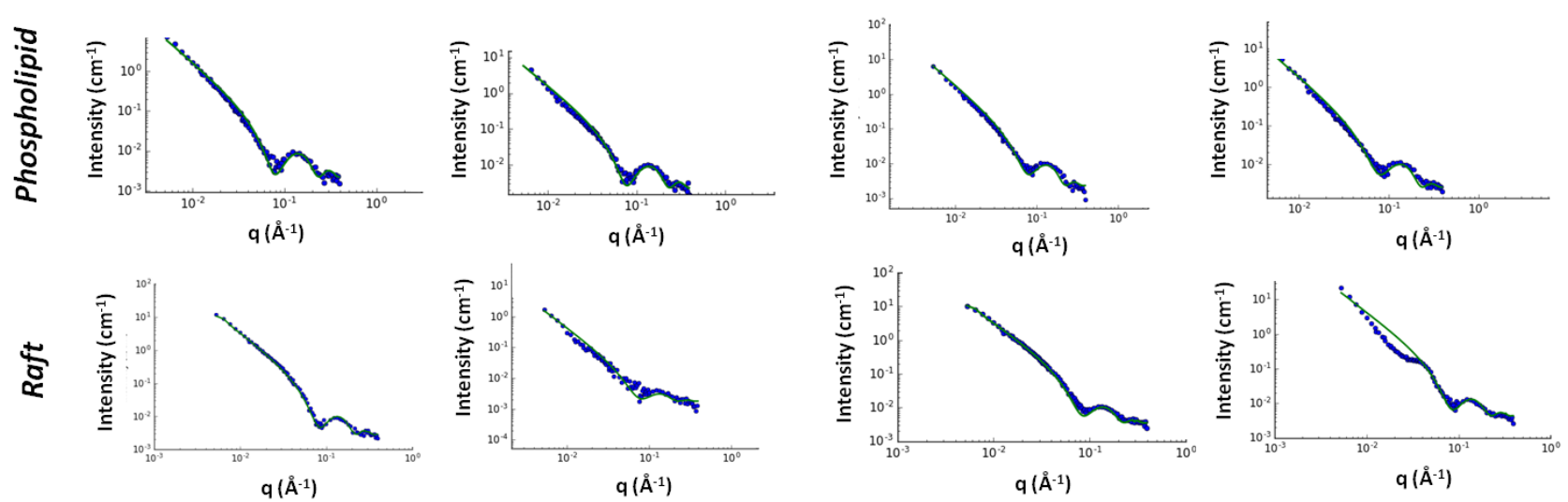
Figure S1. SANS spectra (dots) and best fits (lines) of the vesicle systems (top line:phospholipid only, bottom: phospholipid+GM1) investigated as prepared, in Fe(II) containing solution, and in interaction with the protein at different states of aggregation.

Table 1. Fitting results for the SANS measurements

\begin{tabular}{|c|c|c|c|c|c|c|}
\hline & $\begin{array}{l}\text { SLD heads in } \\
\left( \pm 0.1^{*} 10^{-6} \AA^{-2}\right)\end{array}$ & $\begin{array}{c}\text { Thickness } \\
\text { heads in }( \pm 1 \AA)\end{array}$ & $\begin{array}{l}\text { SLD chains } \\
\left( \pm 0.1 * 10^{-6} \AA^{-2}\right)\end{array}$ & $\begin{array}{c}\text { Thickness } \\
\text { Chains }( \pm 1 \AA)\end{array}$ & $\begin{array}{l}\text { SLD heads out } \\
\left( \pm 0.1 * 10^{-6} \AA^{-2}\right)\end{array}$ & $\begin{array}{c}\text { Thickness } \\
\text { Heads out } \\
( \pm 1 \AA)\end{array}$ \\
\hline DMPC & 1.7 & 7 & 6.3 & 32 & 1.7 & 8.5 \\
\hline $\mathrm{DMPC}_{\mathrm{Fe}}$ & 1.7 & 7 & 6.4 & 32 & 1.7 & 8.6 \\
\hline $\mathrm{DMPC}+\alpha_{\mathrm{mon}}$ & 1.7 & 7 & 6.3 & 31 & 2 & 14 \\
\hline $\mathrm{DMPC}_{\mathrm{Fe}}+\alpha_{\mathrm{oli}}$ & 1.7 & 7 & 6.5 & 29 & 2 & 14 \\
\hline DMPC-GM1 & 1.65 & 7 & 5.6 & 33 & 1.75 & 12 \\
\hline DMPC-GM1 $_{\mathrm{Fe}}$ & 1.65 & 7 & 6 & 32 & 1.75 & 12 \\
\hline DMPC-GM1 $+\alpha_{\text {mon }}$ & 1.67 & 7 & 5.6 & 32 & 1.64 & 13 \\
\hline DMPC-GM1 $1_{\mathrm{Fe}}+\alpha_{\mathrm{oli}}$ & 1.65 & 7 & 5.6 & 32 & 1.75 & 13 \\
\hline
\end{tabular}



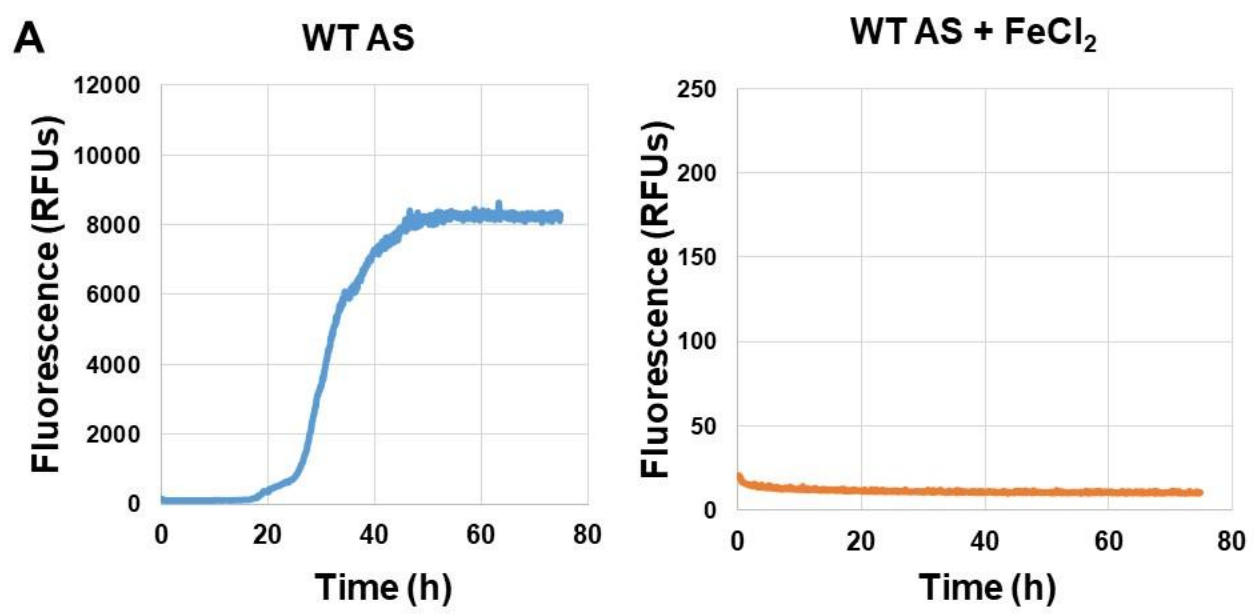

B
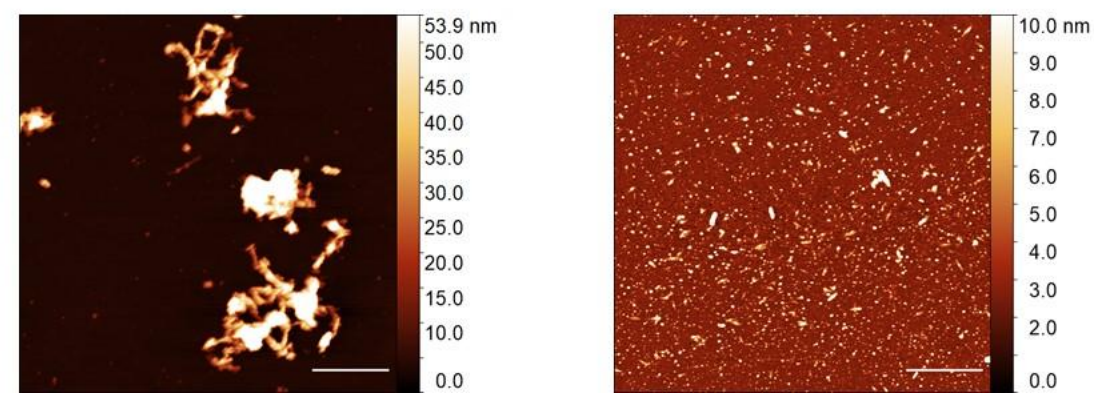

Figure S2. (A) TioflavinT (ThT) assay of wild-type (WT) AS in presence and absence of $\mathrm{FeCl}_{2}$. (B) AFM images of each protein aggregates after 72 hours of incubation. In the absence of iron the aggregation of the protein reaches saturation after 50 hours, displaying the typical sigmoidal curve for the aggregation of amyloid proteins. The AFM image confirms the presence of fibrillar and amorphous aggregates. On the contrary, iron does not induce any significant increase in the fluorescent emission due to the presence of fibrillar and/or beta-rich oligomeric species of AS. AFM analysis confirms the presence of globular aggregates similar to those reported in Figure 1B on page 6, Scale bar: $1 \mu \mathrm{m}$. 